\title{
Validation of the Thyrotoxicosis-associated Insomnia Model Induced by Thyroxine through Sympathetic Stimulation: Face, Construct and Predictive Perspectives
}

\author{
Zhifu $\mathrm{Ai}^{1 \dagger}$, Hongwei He${ }^{1 \dagger}$, Tingting Wang ${ }^{1}$, Liling Chen ${ }^{1}$, Chunhua Huang ${ }^{1}$, Changlian Chen ${ }^{1}$, \\ Pengfei $\mathrm{Xu}^{1}$, Genhua $\mathrm{Zhu}^{1}$, Ming Yang ${ }^{1,2}$, Yonggui Song ${ }^{1 *}$ and Dan $\mathrm{Su}^{1 *}$ \\ ${ }^{1}$ Key Laboratory of depression animal model based on TCM syndrome, Jiangxi Administration of traditional Chinese \\ Medicine, Jiangxi Key Laboratory of TCM for prevention and treatment of brain diseases with cognitive impairment, Jiangxi \\ University of Chinese Medicine, Nanchang 330006, ${ }^{2}$ Key Laboratory of Modern Preparation of Traditional Chinese Medicine, \\ Ministry of Education, State Key Lab of Innovation Drug and Efficient Energy-Saving Pharmaceutical Equipment, Jiangxi \\ University of Traditional Chinese Medicine, Nanchang 330004, China
}

\begin{abstract}
Insomnia has become a common central nervous system disease. At present, the pathogenesis of insomnia is not clear. Animal models can help us understand the pathogenesis of the disease and can be used in transformational medicine. Therefore, it is very necessary to establish an appropriate model of insomnia. Clinical data show that insomnia patients with high levels of thyroxine and often accompanied by cardiovascular problems, a common mechanism underlying all of these physiological disruptions is the sympathetic nervous system. Combined with the characteristics of chronic onset of clinical insomnia, an insomnia model induced by long-term intraperitoneal injection of thyroid hormone has been created in our laboratory. In this paper, the insomnia-like state of the model was evaluated based on three validity criteria. Face validity has been demonstrated in metabolism, the Morris water maze, electrocardiogram (ECG) and electroencephalogram (EEG). Structure validity has been proved by the results of targeted metabolomics. After treatment with diazepam, a commonly used clinical anti-insomnia drug, the above physiological and pathological disorders were reversed. The results of comprehensive analysis show that the established thyrotoxicosis-associated insomnia model meets the validity requirement to establish an appropriate animal model of insomnia. The model presented in this article might help to study pathogenetic mechanisms of clinical insomnia, as well as to test promising methods of insomnia treatment.
\end{abstract}

Key words: Insomnia, Animal model, Thyroxine, Sleep disorders, Rats

\section{INTRODUCTION}

Chronic insomnia is by far the most common sleep disorder in medical practice, which is defined as complaining about difficul-

Submitted June 27, 2021, Revised November 10, 2021,

Accepted November 17,2021

* To whom correspondence should be addressed.

Yonggui Song, TEL: 86-791-87802135, FAX: 86-791-87802135

e-mail: songyonggui1999@163.com

Dan Su, TEL: 86-791-87802135, FAX: 86-791-87802135

e-mail:sud94@aliyun.com

These authors contributed equally to this article. ties in starting or maintaining sleep in the International Classification of Sleep Disorders. Its adverse effects mainly include cognitive and memory disorders, anxiety, depression, fatigue and so on, seriously affecting our normal life. Nowadays, the incidence of insomnia is increasing year by year, and the prevalence rate has reached 15\% since 2016 [1], but its pathophysiology and pathogenesis are not clear, so it is urgent to study and prevent insomnia. In biomedical research, we often rely on the use of animal models to verify experimental hypotheses and clinical hypotheses, in order to more effectively understand the occurrence and development of human diseases and research prevention and treatment measures. If there is no appropriate animal model, it will hinder the understanding of
Copyright ( $\odot$ Experimental Neurobiology 2021.

www.enjournal.org
This is an Open Access article distributed under the terms of the Creative Commons Attribution Non-Commercial License (http://creativecommons.org/licenses/by-nc/4.0) which permits unrestricted non-commercial use, distribution, and reproduction in any medium, provided the original work is properly cited. 
the mechanism of the disease and the discovery of drugs [2]. The main purpose of translational medicine is to break the inherent barriers between basic medicine and drug development, clinical and public health, and establish a direct connection between them, opening up a revolutionary path for the development of new drugs and the research of new drugs and the study of new treatments. The research and development of new experimental animals and animal models is an important tool to promote preclinical drug research and realize translational research.

In the study of insomnia, the neuromodulation and sleep mechanisms of rodents are similar to those of humans $[3,4]$. Because of their practicability and feasibility, rodents have been widely used in the production of animal models of nervous system diseases. Although there are different rodent insomnia models, they lack good translational value. The sleep stress of these animal models is accumulating and sleep is restricted artificially. Although it can be used to study the abnormal effects of drugs on biological rhythm, intestinal flora or energy metabolism caused by sleep deprivation, it cannot evaluate the direct effects of drugs on sleep. The design and selection of a good animal model is one of the key links in the transition of drugs from preclinical basic research to clinical research. Therefore, it is particularly important to establish an insomnia model that is more in line with clinical practice.

The ideal animal model should be able to simulate the main symptoms of insomnia, be sensitive to positive drugs, and provide reference for the clinical application of candidate drugs in terms of drug dosages, action characteristics and side effects. The experimental method used to construct the model should be as simple, efficient, safe and in line with animal experimental ethics. The main problem facing the construction of animal insomnia model is how to make the model animals have a decline in sleep time and quality under the condition of sufficient sleep time and suitable environment.

According to our previous survey, insomnia patients are often accompanied by elevated thyroid levels $[5,6]$. As an important endocrine hormone, thyroxine can affect the functional changes of endocrine system, cardiovascular system and central nervous system. When thyroid hormone metabolism is disordered, insomnialike performance will occur. Therefore, to mimic the symptoms of insomnia observed in patients with hyperthyroidism, we recently developed a translational model, which is based on long-term intraperitoneal injection of thyroxine. At the same time, diazepam, which commonly used in insomnia patients is used as a verification to evaluate the establishment of insomnia symptoms. The model is a chronic exposure method used to simulate the persistent insomnia-like state in rodents. It provides fundamentally new information about the evolution of chronic insomnia and is help- ful to develop new anti-insomnia treatment strategies.

It is worth noting that the insomnia model provides a system to preclinical investigate the molecular underpinnings of these variable behavioral outcomes while maintaining the validity of the model in three important forms [7, 8]. (i) Face validity, assess how effectively the behavioral and neuropathological phenotypes observed under human conditions reproduce; (ii) Construct validity, show the ability of the model to simulate the pathophysiology of the illness; (iii) Predictive validity, evaluate whether drugs used in the treatment of human diseases can reverse symptoms induced in the model.

In this study, the model of insomnia was established by chronic exogenous intraperitoneal injection of thyroxine for the first time. In this paper, the neurobehavior, endocrine, electrocardiogram (ECG), electroencephalogram (EEG) and sleep-related neurotransmitters of the insomnia model were verified, and the changes of corticosterone reflecting the activity of hypothalamuspituitary-adrenal (HPA) axis were measured, and the insomnia model was comprehensively evaluated. Through this evaluation, we can have a deeper understanding of how thyroid hormone metabolic disorders lead to insomnia. At the same time, because the pathogenesis and concomitant symptoms of this model are highly similar to those of clinical patients with chronic insomnia, it can provide a more powerful basis for clinical diagnosis and treatment of insomnia.

\section{MATERIALS AND METHODS}

\section{Animals and thyroxine administration}

Male SD rat (180 220 g, Center for Animal Laboratory Science and Technology, Jiangxi University of Traditional Chinese Medicine, Nanchang, China) were housed in a 12-h light/dark cycle, with lights off at $18: 00$, room temperature of $25 \pm 1^{\circ} \mathrm{C}$, relative humidity $45 \% \sim 65 \%$ and free access to food and tap water. The rats were randomly divided into three groups, the model group, the diazepam group and the control group ( $\mathrm{n}=6$ /group). Through preliminary experiments, it was determined that the modeling time was 4 weeks, and the thyroxine dose was $300 \mathrm{ug} / \mathrm{kg}$. The model group and the diazepam group were administrated intraperitoneal injection with thyroxine (Shanghai yuanye Bio-Technology Co., CAS\#51-48-9, BR, S20192) dissolution in physiological saline containing $0.1 \mathrm{~mol} / \mathrm{ml}$ sodium hydroxide solution, once a day in a volume of $300 \mathrm{ug} / \mathrm{kg}$ at random times during the light phase. At the same time, the control group was injected with the same amount of normal saline intraperitoneally for 28 consecutive days. After modeling, the diazepam group was given diazepam $1 \mathrm{mg} /$ $\mathrm{kg}$ by gavage, and the model group was given the same amount of 


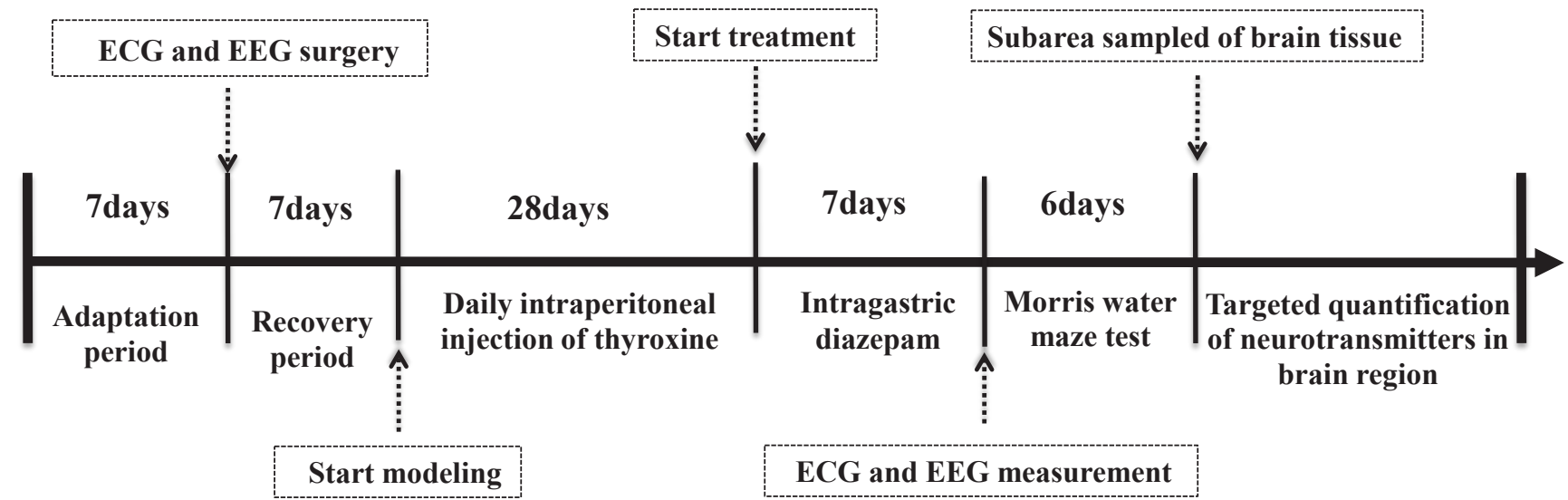

Fig. 1. Time flow chart of experimental design scheme. This figure shows the overall time plan and specific experiment schedule.

normal saline for 7 days.

\section{Study design}

\section{Experimental design}

Before the experiment, all rats were operated on and implanted with wireless transmitter (HD-S11), and one week recovery time was given after the operation. The subsequent intraperitoneal injection of thyroxine lasted for 28 days and diazepam group was perfused by gavage for 7 days, during which daily behavior, weight and diet were assessed. After modeling, Morris water maze test was carried out in each group to evaluate the learning and memory ability. At the same time, ECG and EEG data were collected to evaluate the changes of ECG and EEG. At the end of the experiment, the brain were sampled for targeted metabolomics analysis, and the contents of amino acids and neurotransmitters in brain structure were determined (Fig. 1).

\section{Animal experiments}

\section{Morris water maze test}

The apparatus consists in a circular pool (diameter: $150 \mathrm{~cm}$, height: $50 \mathrm{~cm}$ ) filled with water higher than the platform maintained at $25^{\circ} \mathrm{C}$ and made opaque by addition of white milk powder. The maze was divided into four quadrants (North, South, West, and East). The escape platform was placed in the target quadrant (North), $1 \mathrm{~cm}$ below the water surface during the experiment time. Before the experiment, the rats should be placed in the circular pool for 2 minutes to swim freely, so that the rats can adapt to the environment in the pool ahead of time, at the same time, the individual rats with greater response to water stimulation can be eliminated. During training period, the platform was hidden to develop spatial learning. The rats received 90 s trials (Day 1 5) with 1 minutes inter-trial intervals. The time limit for searching the platform was set to $90 \mathrm{~s}$ as the escape latency. Rats were allowed to stay on the platform for $30 \mathrm{~s}$ after each experiment. If the rats did not find the platform within $90 \mathrm{~s}$, the experimenter should gently guide the animal to the platform. A $120 \mathrm{~s}$ spatial probe test (Day 6), during which the platform was removed, to performed $24 \mathrm{~h}$ after the last trial of the learning period. The escape latency of the experiment, the number of times of $120 \mathrm{~s}$ crossing the platform and the time of $120 \mathrm{~s}$ staying in the platform quadrant were recorded.

\section{EEG and ECG surgery}

The rats were surgically implanted with one of the smallest available wireless transmitters (Ambient Pressure Reference Model APR-1, HD-S11) for recording EEG, ECG and temperature. The animals were free to eat and drink water before the operation, and the animals were anesthetized with a small animal inhaled gas anaesthesia machine equipped with isoflurane liquid. First, the animals were put into the induction anesthesia box to open the anesthetic evaporator to the maximum. After the animals entered the state of deep anesthesia, the animals were taken out, put on the breathing mask connected to the anesthesia machine and lay on the operating table. At the same time, adjusted the tidal volume knob to make the tidal volume meet the needs of the operation. The operating table was disinfected with 75\% ethanol in advance, and the constant temperature heating plate was placed under the back of the animal to maintain a body temperature of about $37^{\circ} \mathrm{C}$ fixed rats, removed their abdominal hair with a razor, disinfected the abdominal skin with iodophor, and iodinated with $75 \%$ (volume fraction) ethanol once; along the midline of the abdomen, cut the skin about $2 \mathrm{~cm}$ at the $1 \mathrm{~cm}$ under the xiphoid process. The body of the telemetry transmitter was implanted into the peritoneum through a median abdominal incision. At the same time, the catheter was inserted into the aorta for about $2 \mathrm{~cm}$ and fixed. 


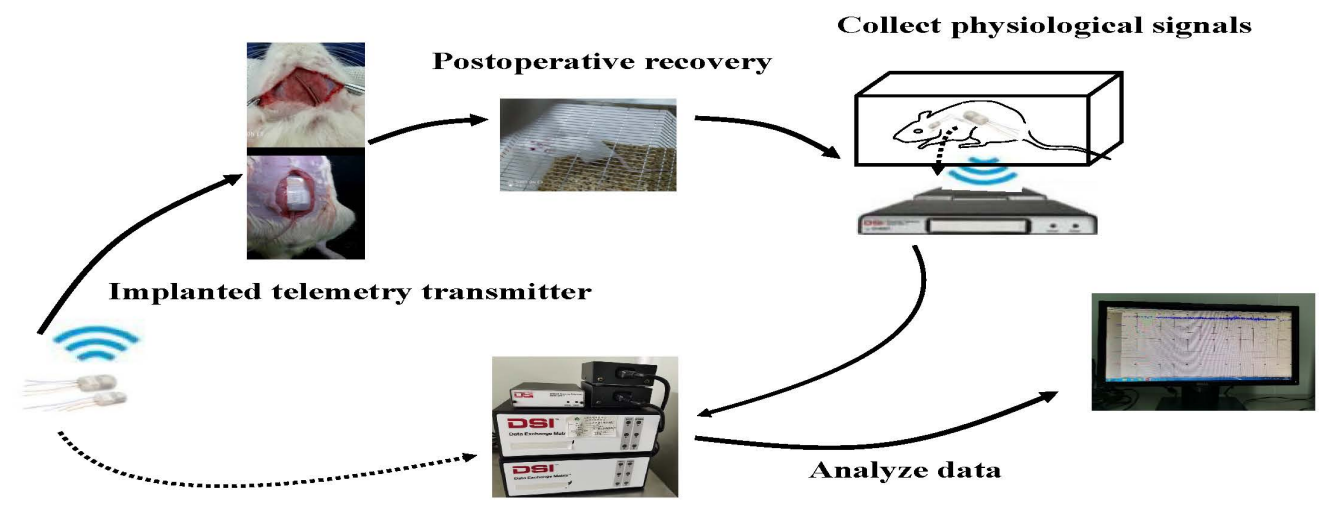

Fig. 2. Flow chart of physiological signal telemetry technology implantation and signal collection. The telemetry transmitter was implanted into the abdomen and skull of rats to record ECG, EEG, body temperature and other related data. Data collection begins after the 7-day recovery period, and the experimental animals remain free of movement. All signals are transmitted wirelessly to the receiver, and the data are processed and analyzed by a matching analysis system. In the figure, the dotted arrow represents the transmission direction of the wireless signal, and the solid line represents the advance of the experimental process.

Guided the lead to the subcutaneous part of the skull and then sutured the abdominal muscle wall and skin incision with simple interrupted-pattern sutures. Holes were drilled in the skull to implant two EEG spiral electrodes (Bregma: A: 1.0, L: 1.0; P: 3.0, L: 3.0 contralateral). Then covered the screw electrode and telemetry line with dental acrylic resin and the scalp was sutured tightly. Iodine disinfectant was used to disinfect surgical wounds. Penicillin sodium should be injected intraperitoneally every day within 5 days after surgery in order to prevent postoperative infection. At the same time, the experiment was carried out 7 days after recovery. The experimental data of 3 days were collected, the first day was to eliminate the interference factors in the environment, and the reliability of the collected data was determined. The data collected on the second day was used for analysis, and the data on the third day was used for data correction (Fig. 2).

\section{Targeted metabolomics quantitative analysis of brain Brain extraction}

At the end of the experiment, rats were executed by neck release. In the meantime, subregional dissection to separate brains into brain stem, cerebral cortex, hypothalamus and hippocampus. Following dissection, all tissues were flash frozen in liquid nitrogen and stored at $-80^{\circ} \mathrm{C}$ until metabolomic analysis.

\section{Preparation of brain}

The frozen brain samples were thawed, then dissected and homogenized in a four-fold excess volume of methanol. The homogenates were centrifuged at $13,000 \times \mathrm{g}$ for $10 \mathrm{~min}$ at $4{ }^{\circ} \mathrm{C}$. Then, the supernatant of $100 \mathrm{ul}$ was transferred to a $10 \mathrm{ml}$ centrifuge tube, the methanol solution of $400 \mathrm{ul}$ was added, and then vortexing for $5 \mathrm{~min}$ and shaking for $5 \mathrm{~min}$. Next, the supernatant of $100 \mathrm{ul}$ was transferred into the $1.5 \mathrm{~mL}$ centrifuge tube together with the IS solution of $10 \mathrm{ul}$ and the methanol solution of $200 \mathrm{ul}$, followed by vortexing for $5 \mathrm{~min}$ and shaking for $5 \mathrm{~min}$. These samples were centrifuged for 10 minutes under the condition of $10,000 \times \mathrm{g}$ and the centrifugation time was $4^{\circ} \mathrm{C}$. The supernatants were quantitatively transferred to a $5 \mathrm{ml}$ glass tube and evaporated to dryness at $35^{\circ} \mathrm{C}$ under a slight stream of nitrogen. Finally, the dried extract was reconstituted in $100 \mathrm{ul}$ acetonitrile solution, followed by the injection of a 2 ul aliquot into the Ultra Performance Liquid Chromatography/Tandem Mass Spectrometry (UPLC-MS/MS) system for analysis [9].

\section{UPLC-MS/MS analysis}

A Shimadzu UPLC system (Shimadzu) was interfaced to an Agilent 5500 QQQ-MS/MS (Agilent Technologies) equipped with an electrospray ionization (ESI) source. The analytes were separated on Agilent C18 column ( $2.1 \times 100 \mathrm{~mm}, 3.5 \mathrm{um})$ with the column temperature set at $40^{\circ} \mathrm{C}$. The mobile phase comprised solvent $\mathrm{A}$ ( $0.1 \%$ formic acid in water) and solvent B (acetonitrile). The mobile phase was eluted at $0.2 \mathrm{ml} / \mathrm{min}$ according to the gradient as follows: $0.001 \min 2 \%$ B; $0.001 \sim 1 \min 2 \%$ B; 1 4 $\min 2 \% \sim 30 \%$ B; 4 10 $\min 30 \% \sim 90 \%$ B; 10 10.1 min 90\% 100\% B; 10.1 13.1 min $100 \%$ B; 13 13.1 min 100\% 5\% B; stopped at 15 min.The injection of a $2 \mathrm{ul}$ aliquoted into the UPLC-MS/MS system for analysis $[10,11]$.

The ESI source was operated in the positive mode, and its main working parameters were set as follows: curtain gas, $35.00 \mathrm{psi}$; lon source gas 1, 45.00; Ion source gas 2, 40.00 ; Ion source temperature, $550.00^{\circ} \mathrm{C}$; entrance potential, $10.00 \mathrm{~V}$; collision chamber 
outlet voltage, $14 \mathrm{~V}$; collision-induced pyrolysis voltage, $7 \mathrm{psi}$. The optimized Multiple Reaction Monitoring (MRM) parameters obtained from the separation analysis in Analyst Software 1.6.2.

\section{Statistical analysis}

Face validity results were evaluated by student's $t$ test; Construct and predictive results were evaluated by Two-way analysis of variance (ANOVA) followed by Tukey's for detection of differences between groups. All statistical tests have been tested for multiple comparisons with Bonferroni correction. Data are represented as means \pm SEM. Software used in analysis was GraphPad Prism 8.3.0. ${ }^{*} \mathrm{p}<0.05$ was required to rate differences as statistically significant, ${ }^{* *} \mathrm{p}<0.01$ and ${ }^{* * *} \mathrm{p}<0.001$ were the very significant difference.

\section{RESULTS}

\section{Face validity of the animal model of thyrotoxicosis-associated insomnia induced by thyroxine}

The face validity of thyrotoxicosis-associated insomnia disorder animal model implies compliance with the model based on its behavioral manifestations. It can be observed that these animals showed an increase in metabolism and a decrease in learning and memory ability. According to the analysis of the results in Fig. 3, he weight gain of the model group decreased significantly (Fig. 3A), but the food intake increased significantly (Fig. 3B), so the Diet/ weight in the model group was significantly higher than that in the control group (Fig. 3C), and the difference gradually increased with the increase of modeling time. In the early training of the water maze, the escape latency of the model group was higher than that of the control group (Fig. 3D). On the sixth day, the spatial probe test was carried out to evaluate short-term spatial memory. Compared with the control group, the time of staying in the target quadrant and the number of crossing the platform in the model group were significantly less than those in the control group (Fig. $3 \mathrm{E}, 3 \mathrm{~F})$. At the same time, the trajectory map and hot zone map showed that the remotecontrol range of the control group was more concentrated in the target quadrant (Fig. 3G). The analysis of the results of EEG showed that the hypnogram of control group was different from that of model group, indicating that injection of thyroxine could change the sleep habits of rats. At the same time, the total duration of Non-Rapid Eye Movement (NREM) sleep in model group was significantly shortened, and the total duration of awake was significantly increased (Fig. 4A, 4B). The spectral analysis showed that long-term injection of thyroxine had an effect on EEG rhythm. From the power spectrum distribution results of each group, it can be seen that the dominant frequencies of each group are distributed in the delta with lower frequency, and there is a significant difference between the blank group and the model group. At the same time, in the theta frequency band and the alpha frequency band, the frequency percentage of the model group is higher than that of the control group (Fig. 4C). In the REM stage, the model group showed a decrease in the delta rhythm activity level and a significant increase in the alpha rhythm of the highfrequency rhythm activity (Fig. 4D). At the same time, during the NREM stage, the delta rhythm activity level was also decreased and the beta rhythm activity level increased significantly (Fig. 4E), suggesting that the rats in the model group had excessive arousal. Analysis of the duration of sleep/wake episodes revealed that in the dark phase (08PM 07AM), the wake time and REM time of the model group were prolonged, while the NREM time was significantly decreased. In the light phase (08AM 07AM), the REM time of the model group was significantly increased, but there was no significant difference between the awakening time and the NREM time (Fig. 4F 4H). From the change of 24 -hour body temperature, the body temperature of the model group was higher than that of the control group during sleep (Fig. 4I). In order to evaluate the effect of chronically elevated thyroxine on the cardiovascular system, ECG parameters were measured. ECG-related results showed that PR interval and RR interval were shortened and HR was increased in the model group, indicating the symptoms of tachycardia in the model group (Fig. 4J 4L). Heart Rate Variability (HRV) is a commonly quantitative indicator to determine the function of the cardiac autonomic nerve, Low Frequency/ How Frequency (LF/HF) with heart rate variability can reflect the balance regulation between the sympathetic nerve and the vagus nerve to express the regulating ability of the autonomic nervous system. When LF/HF increased, it showed that the balance between sympathetic nerve and vagus nerve decreased, the balance regulation ability of autonomic nervous system decreased, and sympathetic nerve was relatively excited. It turns out that the HRV of the model group was apparently higher than that of the control group during sleep (Fig. 4M), indicating that the sympathetic activity of the model group was enhanced, and that there was the excessive awakening of physiological activities. Together, these results demonstrate that long-term injection of thyroid hormone induces insomnia-like behavior in the same rat, contemplating the face validity for a fit animal model of insomnia.

\section{Construct validity of the animal model of thyrotoxicosis-associated insomnia induced by thyroxine}

The construct validity of the animal model suggests that the modeled insomnia features should be unambiguously interpreted and regarded as homologous, and should be related to insomnia both empirically and theoretically. To evaluate the structural validi- 
A

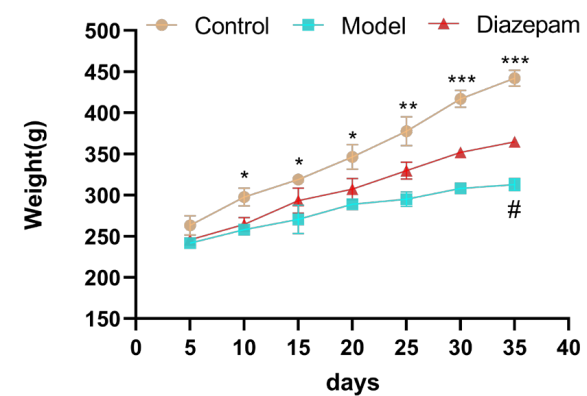

C

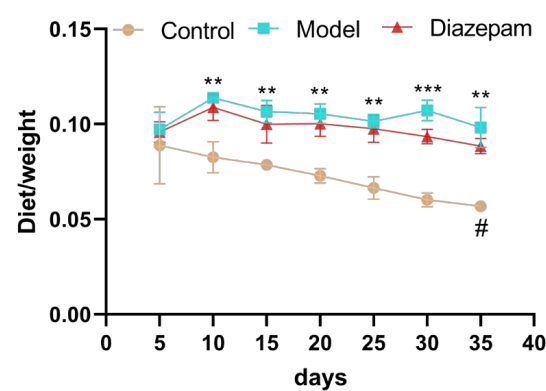

E

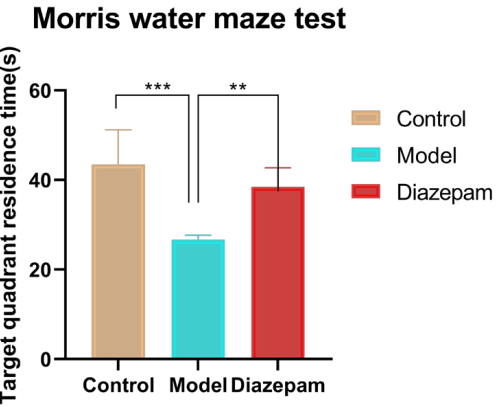

B

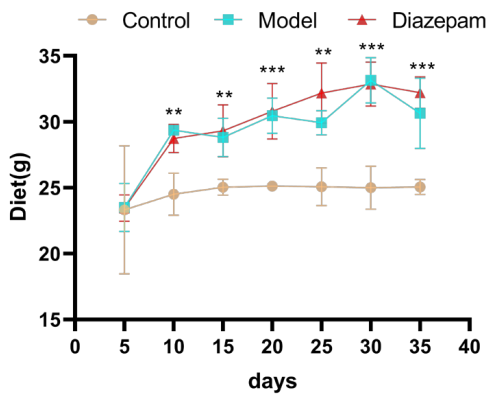

D

Morris water maze test

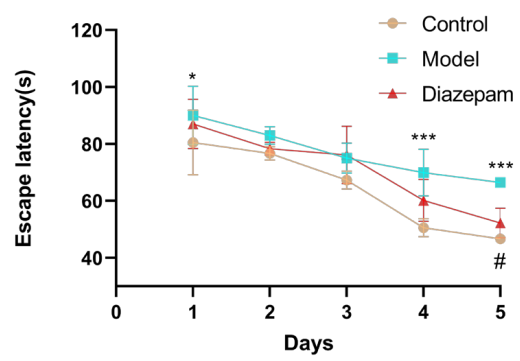

F

Morris water maze test

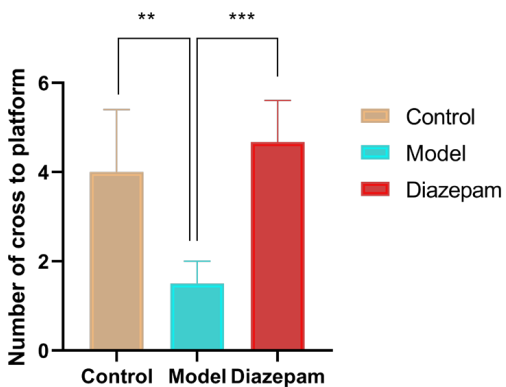

G

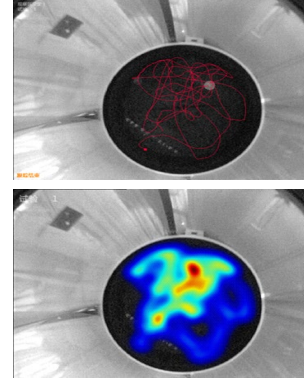

Control

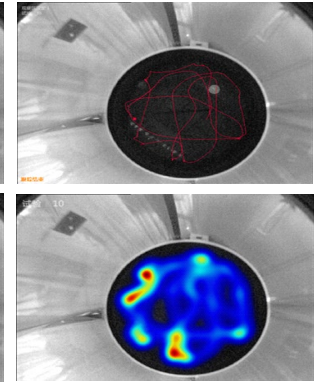

Model

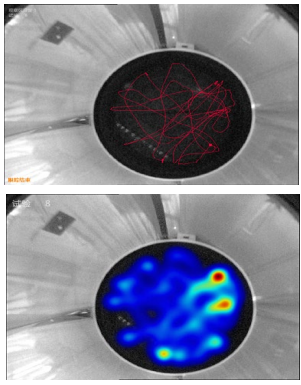

Diazepam

Fig. 3. Effects of thyroxine on face validity after chronic exogenous intraperitoneal injection in rats. (A) Mean body weight during the stress; (B) Diet during the stress; (C) Diet/weight during the stress; (D) Located voyage escape latency in experimental rats (Day 1 5); (E) Target quadrant residence time of experimental rats in space exploration experiment (Day 6); (F) The number of cross to platforms in the space exploration experiment of experimental rats (Day 6); (G) The trajectory map and hot zone map of rat space exploration experiments; Data are represented as means \pm SEM, ${ }^{*} \mathrm{p}<0.05$, ${ }^{* *} \mathrm{p}<0.01$, ${ }^{* * *} \mathrm{p}<0.001$ were considered to indicate significant difference between Control and Model. ${ }^{*} \mathrm{p}<0.05,{ }^{\# *} \mathrm{p}<0.01$ were considered to indicate significant difference between Diazepam and Model. 
A

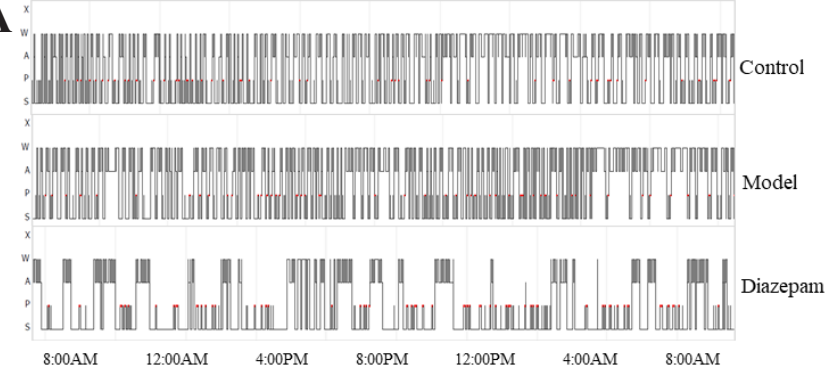

C

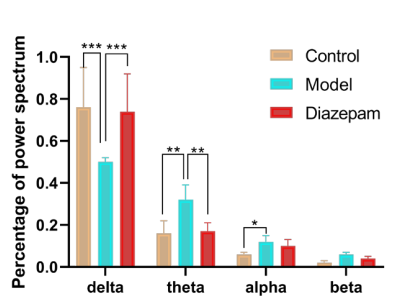

D

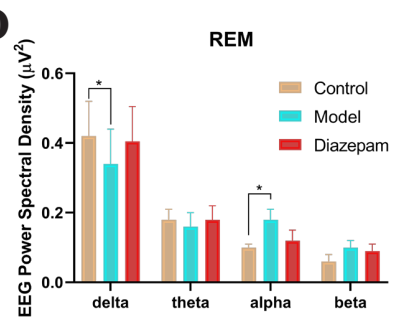

B

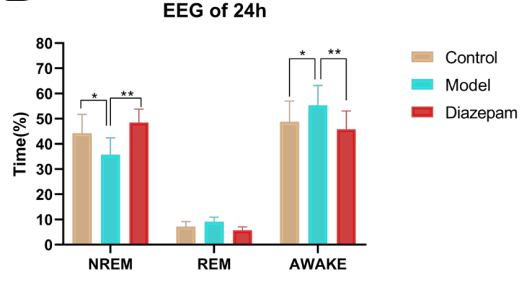

E

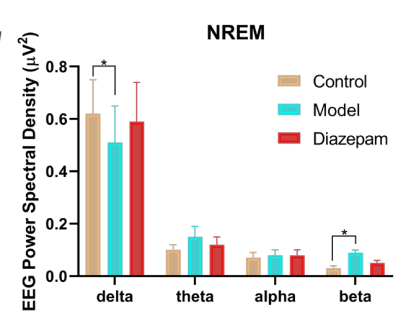

H

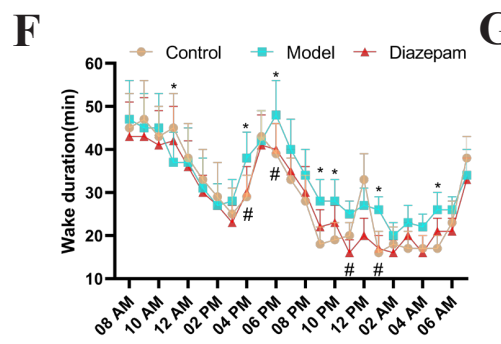

G
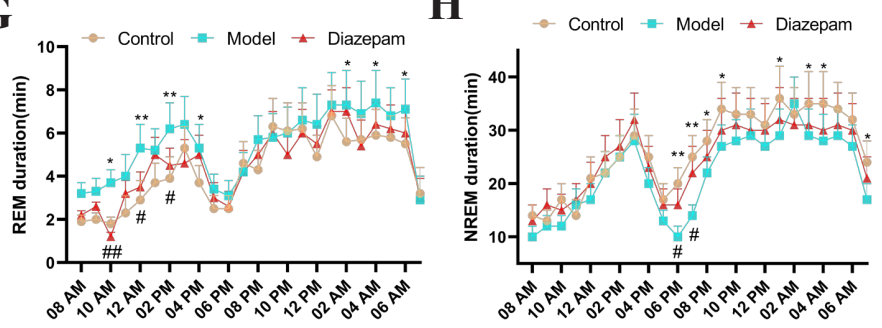

I

$\mathbf{J}$
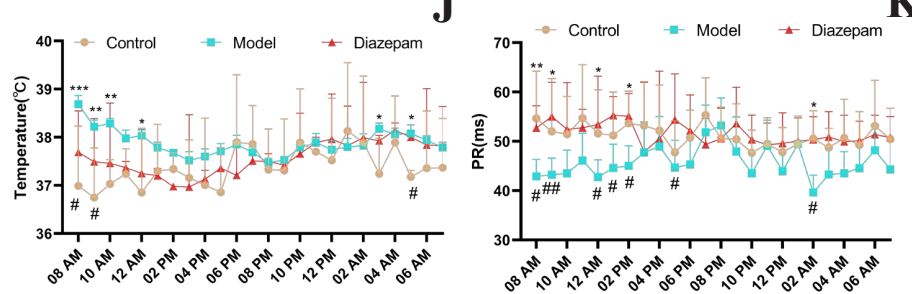

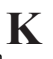

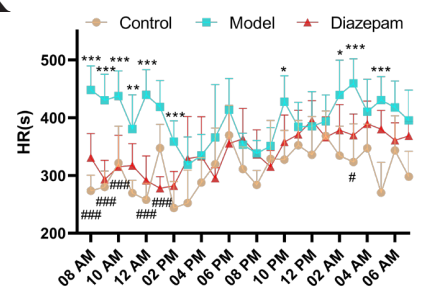

L
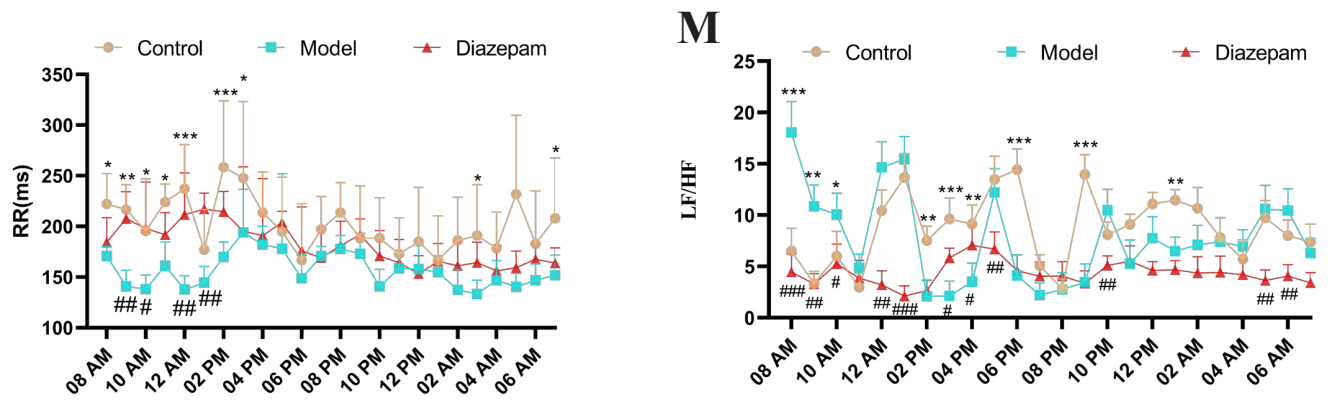

Fig. 4. Effects of thyroxine on EEG and ECG after chronic exogenous intraperitoneal injection in rats. (A) Representatives hypnograms of control, model and diazepam groups. (B) The effect of chronic thyroxine disorder on 24-hour sleep awakening patterns. (C) Effect of chronic thyroxine disorder on the percentage of power spectrum distribution. (D) Total EEG spectral density of each frequency band in REM period. (E) Total EEG spectral density of each frequency band in NREM period. (F) Recording 24-hour continuous wake duration (minutes) changes. (G) Recording 24-hour continuous REM duration (minutes) changes. (H) Recording 24-hour continuous NREM duration (minutes) changes. (I) Effect of chronic thyroxine on body temperature of experimental rats. (J) Recording 24-hour continuous PR changes. (K) Recording 24-hour continuous HR changes. (L) Recording 24-hour continuous RR changes. (M) 24-hour changes of LF/HF in heart rate variability. Data are represented as means $\pm S E M,{ }^{*} p<0.05,{ }^{* *} p<0.01$ and ${ }^{* * *} p<0.001$ were considered to indicate significant difference between Control and Model. ${ }^{*} \mathrm{p}<0.05,{ }^{* *} \mathrm{p}<0.01,{ }^{\# * *} \mathrm{p}<0.001$ were considered to indicate significant difference between Diazepam and Model. W, Wake; A, Active Wake; P, Paradoxical sleep; S, Slow-Wave Sleep; REM, Rapid Eye Movement; NREM, NonRapid Eye Movement; HR, Heart Rate; PR, the P-R interval, from the beginning of P wave to the beginning of R wave in QRS complex in ECG; RR, the distance between R wave and R wave on ECG; LF, Low Frequency in heart rate variability; HF, High Frequency in heart rate variability. 
ty of an animal model of insomnia induced by long-term injection of thyroid hormone, we evaluated the changes of biomarkers observed in patients with insomnia, such as melatonin receptor, HPA axis, and insomnia-related neurotransmitters. The well-known function of melatonin is to improve the latency, duration, and quality of sleep, and focus on the maintenance of circadian rhythm and sleep regulation. These effects are mainly accomplished by feedback from the hypothalamic suprachiasmatic nucleus, so we analyze the expression of melatonin receptors in the hypothalamus by immunofluorescence technique. The results show that the expression of melatonin receptors in the model group is significantly lower than that in the control group (Fig. 5A, 5B). HPA axis plays an important role in regulating the changes of Sleep, in which awakening is closely related to the increase of corticosterone levels. In this experiment, the concentration of corticosterone in each brain region of the model group increased in varying degrees (Fig. $5 \mathrm{C})$, especially in the cerebral cortex and brainstem. The changes of neurotransmitters and amino acids in brain structure can reflect the corresponding neuronal activity. Therefore, we measured the changes of neurotransmitters and corresponding amino acids in brain regions to understand the specific biological function of chronic thyroxine stress. Fig. 5D shows that the concentration of 5-hydroxytryptamine (5-HT) in the brain stem and hippocampus of model groups increases significantly, it is suggested that chronic thyroxine imbalance can increase the activity of serotonin neurons in the brain stem and hippocampus. 5-HT, glutamic acid (Glu), $\gamma$-aminobutyric acid (GABA), and other neurotransmitters and amino acids are closely related to sleep-awakening process. After long-term injection of thyroid hormone, the concentration of 5-HT in the brain stem and hippocampus of model rats increased significantly (Fig. 5D), and the concentration of Glu in the cerebral cortex and hippocampus also increased significantly (Fig. 5E). At the same time, the concentration of GABA decreased significantly in the cerebral cortex, brain stem, hippocampus, and hypothalamus (Fig. 5F). Together these results demonstrate that long-term injection of thyroid hormone has construct validated because the concentrations of neurotransmitters and amino acids in different brain regions observed in patients with insomnia change.
A
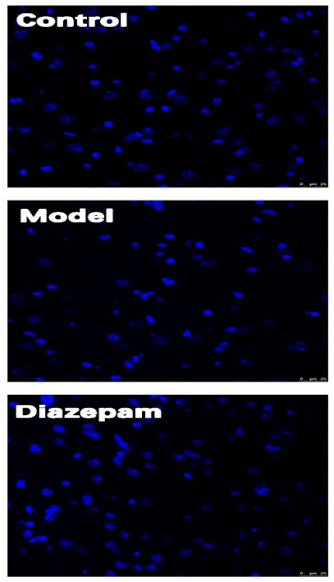

C

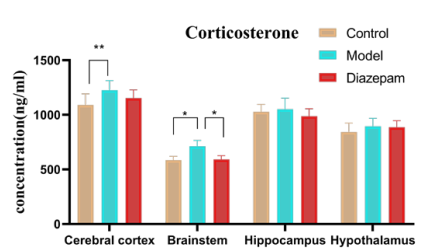

MT
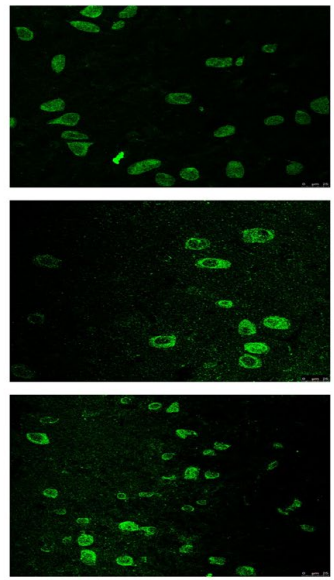

D

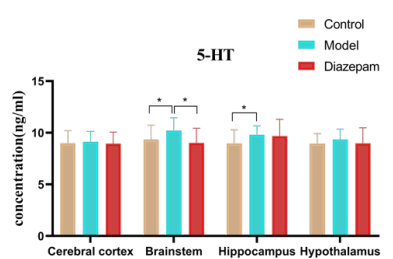

Merge
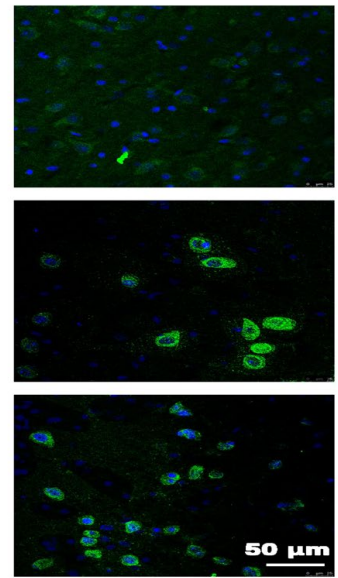

E

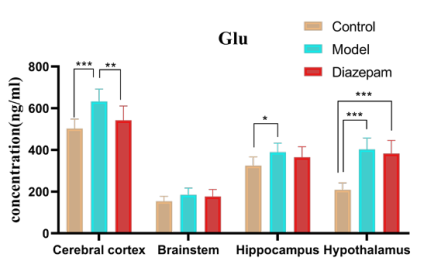

B

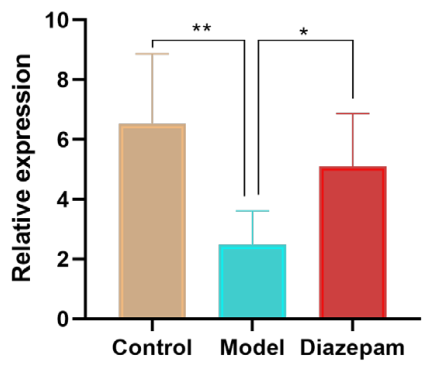

Fig. 5. Effects of thyroxine on construct validity after chronic exogenous intraperitoneal injection in rats. (A, B) Effect of chronic elevation of thyroxine on the expression of melatonin receptor in hypothalamus of experimental rats. Immunofluorescence assay of MT (green) and DAPI (blue) expression in hypothalamus. Scale bar: $50 \mu \mathrm{m}$. (C) Distribution of corticosterone in the structure of functional brain regions. (D) Changes of 5-HT concentration in different brain regions. (E) Changes of Glu concentration in different brain regions. (F) Changes of GABA concentration in different brain regions. Data are represented as means $\pm \mathrm{SEM},{ }^{*} \mathrm{p}<0.05,{ }^{* *} \mathrm{p}<0.01,{ }^{* * *} \mathrm{p}<0.001$ were considered to indicate significant difference between Control and Model. MT, melatonin receptor; DAPI, 4,6-diamidino-2-phenylindole; 5-HT, 5-hydroxytryptamine; Glu, glutamic acid; GABA, $\gamma$-aminobutyric acid. 


\section{Predictive validity of the animal model of thyrotoxicosis-associated insomnia induced by thyroxine}

For the model of insomnia-like behavior in animals, the predictive validity primarily means a response to anti-insomnia therapy. As described previously in the Figure, the weight of rats treated with diazepam increased significantly (Fig. 3A), but there was no significant change in diet (Fig. 3B), so the Diet/weight in the diazepam group was slightly lower than that in the model group (Fig. 3C). At the same time, the daytime body temperature of the diazepam group decreased significantly (Fig. 4I), indicating that its metabolic ability decreased. In the early training of the water maze, the escape latency of the diazepam group was lower than that of the model group, especially on the 4th and 5th day, there was a significant difference between the two groups (Fig. 3D). Compared with the model group, the diazepam group stayed more time in the target quadrant and crossed the platform more often (Fig. 3E, 3F). At the same time, the trajectory map and hot zone map showed that the remote range of the diazepam group was more concentrated in the target quadrant (Fig. 3G). Such alterations in REM, NREM, and AWAKE sleep parameters may provide useful data about outcomes of certain therapeutics. After treatment, the total duration of NREM sleep in the diazepam group was significantly increased, the total duration of REM sleep had no significant change, and the total duration of awake was significantly shortened in the diazepam group (Fig. 4A, 4B). The results of the spectral analysis showed that the percentage of delta band increased significantly and the percentage of theta band decreased significantly after diazepam treatment (Fig. 4C). In REM and NREM stages, delta, theta, and alpha all showed a tendency to pull back, but there was no significant difference (Fig. 4D, 4E). The awakening time of rats decreased significantly in the dark phase, and there was no significant change in REM and NREM time. In the light phase, only REM time increased significantly (Fig. 4F 4H). The related parameters of ECG showed that diazepam decreased the accelerated heart rate caused by a long-term injection of thyroid hormone in the model group and effectively slowed down the tachycardia in the model group (Fig. 4J 4L). From the results of HRV (Fig. 4M), it can be seen that diazepam can reduce the heart rate variability of rats, especially during sleep. The results of immunofluorescence analysis showed that the expression of melatonin receptors in the diazepam group was significantly higher than that in the model group (Fig. 5A, 5B). From the results of the changes of corticosterone concentration in each brain region (Fig. 5C), it can be seen that the corticosterone concentration decreased in the rats treated with diazepam, especially in the brainstem. After treatment with diazepam, the concentration of neurotransmitters in all brain regions changed, the concentration of 5-HT in the brain stem decreased significantly (Fig. 5D), and the concentration of glutamate in the cerebral cortex decreased significantly (Fig. 5E). It is worth noting that the concentration of GABA in the cerebral cortex, brainstem, and hypothalamus increased significantly (Fig. 5F). Therefore, to sum up, it can be considered that diazepam has a therapeutic effect on thyroxine-induced thyrotoxicosis-associated insomnia disorder in rats, contemplating the predictive validity of the model.

\section{DISCUSSION}

No single animal model can account for the entire disease it purports to represent. Therefore, the validation criteria that each model is expected to fulfill in order to demonstrate its validity are, for practical purposes, largely determined by the objective of the model and its intended use. Simulating specific behavioral symptoms can help us understand the pathogenesis of the disease and can be used in translational research. In this experiment, we established the thyrotoxicosis-associated insomnia model to fully simulate the chronic development process and pathophysiological changes of clinical insomnia patients, and provide a more effective and less side effects diagnosis and treatment scheme for clinical insomnia patients. On the one hand, attention should be paid to the observation and analysis of animal behavior and general state in the research, and a variety of analytical indicators should be established to reproduce the clinical manifestations of insomnia as completely as possible. Reliability, on the other hand, reliability requires that the behavior of the model is effective and repeatable. Finally, concerning predictive validity, anti-insomnia drugs, such as diazepam, have been shown to improve the symptoms of insomnia.

Insomnia is a kind of organic insomnia syndrome, which is related to the dysfunction of neuronal circuits involved in sleep regulation and excessive activation of sympathetic nerves [12-15]. A large number of cross-sectional case-control studies have found that increased activity of the HPA axis (such as corticosterone) [16-18] and autonomic nervous system (such as heart rate and heart rate variability) [19-21] can indicate an increase in the level of arousal. As everyone knows, Insomnia is also closely related to the neuromodulation of the sleep awakening cycle, mainly through the interaction of neurons in the brain circuit that systematically release neurotransmitters. Its neurotransmitters include the awakening neurotransmitters serotonin [22] and glutamate, and the sleep-stimulating neurotransmitter $\gamma$-aminobutyric acid [23].

After studying the long-term clinical observation and empirical findings of insomnia, it is found that insomniacs show signs of in- 
creased arousal at the behavioral, cognitive, autonomic, or central nervous system level [24-26]. The results of various measurements of physiological arousal in patients with insomnia also support that hyperarousal is the main pathophysiological mechanism of insomnia $[27,28]$. In some studies, insomnia is also associated with body temperature [29], metabolic rate [27, 30, 31], heart rate [32] and corticosterone levels [33]. At the same time, a large number of studies have reported an increase in body temperature, metabolic rate, heart rate, and corticosterone concentration in patients with chronic insomnia. To sum up, this abnormal performance is consistent with the state of high physiological awakening [34]. A common mechanism behind all these physiological disorders is the sympathetic nervous system [35]. All these physiological disorders are closely related to the sympathetic nervous system.

Thyrotoxicosis is often associated with symptoms resembling sympathetic activation and psychiatric illness, such as a manic episode of bipolar I disorder. A decreased need for sleep is one of the common symptoms of manic episodes. In addition, the increased number of serotonin neurons may support the possibility of manic change in the mood state [36]. Therefore, before the formal experiment, we need to make it clear that these animals are models of insomnia rather than mania. First of all, the main manifestations of mania can include hyperactivity, emotional elevation or excitement, irritability, reckless behavior, and little need for sleep. In the preliminary experiments, we conducted behavioral tests including open field test and elevated plus-maze test, and there was no significant difference between the model group and the control group, so we did not discuss it. If the animal model is manic, its open field test and elevated plus-maze test will show high activity and desire to explore. At the same time, it is reported that $[37,38]$ the basis of mania is excessive dopamine, and the results of quantitative analysis of targeted metabolomics of the brain showed that there was no significant change in dopamine concentration in the model group. Therefore, we did not discuss this result in the article. Combined with the above experimental results, it can be inferred that the model does not belong to the manic state model.

Thyroid hormones play an important role in nervous system development, energetic metabolism, and thermogenesis. At the same time, it also regulates metabolism and the cardiovascular system [39]. The circadian rhythm disorders of insomnia mainly include delayed or early body temperature rhythm and average body temperature increase during sleep [40]. In order to explore the effect of long-term injection of thyroxine on insomnia model, we evaluate the sleep changes of animal model. It is worth noting that most studies have identified hyperarousal by comparing the differences between the model group and the control group, rather than basing on a specific threshold [27]. First of all, we evaluated the behavior and found that the metabolic ability and body temperature increased in the model group after long-term injection of thyroxine, In the meantime, the cognitive learning ability of the organism was weakened, and the above behavior was reversed after diazepam treatment. It has been proved that thyroxine has an important effect on cardiovascular function [31], and changes in cardiovascular function will also affect sleep regulation [41, 42], at the same time, the circadian changes of sleep are closely related to the regulation of the autonomic nervous system. Therefore, we can explore the effect of long-term injection of thyroid hormone on sleep through the changes of ECG parameters. Among them, the LF/HF of ECG parameter heart rate variability represents the activity of the sympathetic nerve and vagus nerve. According to the results of ECG analysis, it is suggested that the increase of thyroxine level can enhance sympathetic activity, affect the balance of the autonomic nervous system, and accelerate the process of atrial depolarization, ventricular repolarization and cardiac cycle. For the cardiovascular system, LF/HF with heart rate variability could reflect the balance regulation between the sympathetic nerve and vagus nerve to express the energy-saving ability of the autonomic nervous system. Therefore, it reflected from awakening to deep sleep sympathetic nerve excitability was relatively weakened, and vagal nerve excitability was relatively enhanced. From the LF/HF results of heart rate variability and heart rate parameters, it can be seen that the model rats showed a more excited states during sleep (daytime), but relatively quiet during awakening (night), and sleepawakening rhythm was disrupted. After treatment with diazepam, the heart rate and heart rate variability decreased significantly during sleep, suggesting that the excited state of the sympathetic nerve was alleviated. Combining the results of ECG parameters, it can be concluded that thyroxine imbalance can accelerate cardiac repolarization, increase myocardial contractility, affect cardiovascular function, and eventually cause insomnia through sympathetic nervous system mediation, which can be improved by diazepam.

There is evidence that $[33,43,44]$ patients with insomnia, especially those with short objective sleep time, have increased secretion of corticosterone, suggesting increased activity of the HPA axis. In the study of the changes of HPA axis in patients with insomnia, the majority of studies demonstrate that $[33,45,46]$ the activity of HPA axis was overactive in patients with insomnia, indicating that the activity of HPA axis was closely related to insomnia sensitivity. In our study, the concentration of corticosterone in the brain of model rats increased, especially in the brainstem and cerebral cortex, and decreased significantly after administration of diazepam, indicating that long-term injection of thyroid hormone could increase the concentration of corticosterone in brain and increase the activity of HPA axis, and this change could be reversed 
by diazepam. Then, through the analysis of the expression of melatonin receptors in the hypothalamus structure, it was found that the expression of melatonin receptors in the hypothalamus structure of model rats decreased, which indicated that the brain's utilization of melatonin decreased and the sleep-promoting ability was decreased. It has been reported that [47] the levels of melatonin is relevant to sleep-wake regulation. Melatonin is a hormone produced by the pineal gland, which is related to circadian rhythm, mainly through the hypothalamic suprachiasmatic nucleus to regulate the circadian rhythm process in the body. Similarly, the expression of melatonin receptors in the hypothalamus increased after diazepam treatment.

In order to further understand the changes of sleep awakening in the body, we further evaluate the role of thyroxine from the changes of EEG. Hyperarousal has been examined by using various EEG measures during sleep and wakefulness. The increase of REM is an indicator of hyperarousal. A large number of literatures have used the sleep parameters obtained by EEG to evaluate the sleep of patients with insomnia and healthy people with good sleep. The results of the meta-analysis showed that slow-wave sleep and NREM sleep decreased in patients with insomnia [48]. Steiger A [49] research shows that patients with hyperthyroidism are often accompanied by sleep disorders, such as difficulty in starting and maintaining sleep, and a reduction in slow-wave sleep. The EEG of this experiment illustrates that the chronic increase of thyroxine level shortens the sleep time of NREM and prolongs the awakening time of sleep in experimental rats, and substantial electrophysiological effects of diazepam on sleep length seems to be effective enough, in addition to prolonging total sleep time, it can also significantly increase NREM sleep time. As a common resting state EEG data, sleep EEG has made great progress in both medicine and engineering [50]. Collecting sleep EEG signals, analyzing their power spectrum characteristics, and exploring the distribution and activity of EEG in various frequency bands can objectively and effectively reflect brain activity and sleep state [51]. After modeling, the EEG signal is collected, and its time domain characteristics and power spectrum characteristics are analyzed, so that the change of EEG rhythm can be studied. Therefore, using this EEG analysis method as a research parameter to evaluate sleep quality and EEG rhythm changes has a certain degree of universality, international recognition, and greater literature support and reference value. The current study found that the increase of beta rhythm activity and the decrease of low-frequency rhythm activity (delta, theta, and alpha) in insomnia patients at the beginning of sleep or NREM sleep stage suggested that there was excessive awakening in the cerebral cortex of insomnia patients $[52,53]$. In the REM stage, insomnia patients showed a decrease in delta and theta rhythm activity and a significant increase in high-frequency rhythm activity [54]. After injection of thyroxine into rats, the proportion of power spectrum in the delta band of the model group was significantly lower than that of the control group, the theta band was significantly higher than that of the control group, and the alpha, beta band of high frequency band was also relatively higher. The results showed that after the establishment of the model, the main peak of power shifted to the right and the frequency of center of gravity increased, which showed not only the loss of slow-wave sleep, but also the excessive awakening of the cerebral cortex during NREM and REM sleep. Therefore, to sum up, it may be suggested that the effect of long-term intraperitoneal injection of thyroid hormone fully accords with the criteria of thyrotoxicosis-associated insomnia face validity, mimicking not only a series of manifestations such as the hyperarousal state of insomnia patients but also a sympathetic overexcitation observed in insomnia patients.

Different brain regions in the brain could be responded to different physiological functions, and their physiological characteristics are mainly produced by the activity of neurons in corresponding brain regions. Specifically, changes in physiological activities of insomniacs during sleep-awakening can be observed in the following brain regions [55-58]: brainstem, hypothalamus, hippocampus, and cerebral cortex, which maintain stability of sleep awakening through the comprehensive regulation of its neuronal system. At the same time, the cerebral cortex was also the largest part of the brain related to complex brain functions (such as thinking and action) and the hippocampus was an important part related to emotional response. The nervous system originated from the brainstem and the hypothalamus constitutes the ascending reticular activation system, which is closely related to the arousal system and the hyperactive brain. Studies have shown that GABA, Glu, and 5-HT are closely related to sleep [59-61]. GABA is an inhibitory neurotransmitter widely found in the central nervous system, which can inhibit nervous system neurons, has neuroprotective effects and the functions of hypnosis, sedation, anti-anxiety and so on. Glu is an important excitatory amino acid transmitter in the brain, which acts on postsynaptic receptors and promotes the activity of cortical cells to maintain arousal. 5-HT, as an important part of the uplink activation system, plays an important role in maintaining awakening and vigilance. Evidence from various experiments shows that 5-HT neurons promote wakefulness and are a component of the ascending reticular activating system. Similarly, many literatures show that 5-HT promotes sleep. Both sides have an experimental and theoretical basis, and our experimental results prefer that the main function of 5-HT is to promote arousal in thyrotoxicosis-associated insomnia model. It is reported that there are physiological and neurochemical changes in patients 
with insomnia. Therefore, a suitable animal model of insomnia should present this alteration. In the present study, we comprehensively monitored the levels of neurotransmitters in different brain regions of rats to explore the effects of chronic thyroxine stress on the main neurons in the brain region of insomnia model. The results of targeted metabolomics showed that the activity of serotonin neurons, glutamate neurons and other excitatory neurons in cerebral functional areas such as the cerebral cortex, brainstem, hippocampus, and hypothalamus were relatively enhanced, while the activity of aminobutyric acid neurons was relatively weakened. The excitability and awakening ability of the central nervous system were enhanced. It is suggested that long-term intraperitoneal injection of thyroxine may lead to insomnia by enhancing the activity of excitatory neurons in the corresponding functional brain regions. After treatment with diazepam, the concentrations of serotonin and glutamate in each brain region decreased, while the concentration of GABA increased, suggesting that diazepam may improve insomnia by adjusting sleep-related neurotransmitters in the brain. Once again, these research results support the animal model of insomnia to achieve the desired purpose, in line with the face, construct, and predictive validities required to a suitable animal model for insomnia. Data provided by the present research show the insomnia-like changes of rats injected with thyroxine, mimicking pathophysiological aspects in insomnia patients.

All in all, the data suggest that long-term use of thyroxine can constitute a suitable model of insomnia because injection of the drug can cause insomnia-like behavior in the same animal. This modeling method, like other chemical reagent modeling methods, has many shortcomings. Its pathological changes involve multiple organ systems, such as the heart, blood vessels, adipose tissue, muscles, and bones. Due to the extensive impact of thyroid hormones on various body functions, It is hard to rule out the decreased NREM sleep was confounded by the effects on other organ systems. Although we are unable to clarify the specific mechanism of the occurrence and development of related insomnia symptoms such as NREM sleep reduction in this model, we experimentally verify that the insomnia of rats after thyroxine injection does exist, which reproduces the significant physiological and neurochemical changes of insomnia patients, including increased metabolism, heart rate variability, sleep cycle changes, HPA axis, and brain neurotransmitters. Therefore, the insomnia animal model based on long-term intraperitoneal injection of thyroid hormone meets the requirements of three primary criteria (validities). It can be used to study the pathophysiology of insomnia and to screen promising candidates for mood stabilizers. In essence, because this model is more consistent with the slow onset of clinical chronic insomnia and is often accompanied by elevated thyroid hormones, this in- somnia model not only improves animal welfare, and may help to promote clinical insomnia research and drug development. Therefore, in view of the current problems regarding the efficiency of preclinical treatment and the challenge of proposing new and effective anti-insomnia drugs, the model of insomnia made by longterm injection of thyroid hormone proposed here is studied by selectively simulating the hormone changes of clinical insomnia patients, which may have more advantages than other translation methods in the study of insomnia.

\section{ACKNOWLEDGEMENTS}

This research was financially supported by National Natural Science Foundation of China (No. 82060808, No. 81860702), Science and technology project of Jiangxi Administration of traditional Chinese Medicine (2021Z011), Jiangxi Provincial Natural Science Foundation (No. 20202BABL206142, No. 20212BAB206012), Establishment project of Jiangxi science and technology innovation platform (No. 20212BCD42009), Science and Technology Key Project of Jiangxi Provincial Department of Education (GJJ201206, GJJ201239), Science and technology project of traditional Chinese medicine in Jiangxi Province (202131057).

\section{REFERENCES}

1. Shao Z, Xu Y, Chen L, Wang S, Zhang M, Liu S, Wen X, Yu D, Yuan K (2020) Dysfunction of the NAc-mPFC circuit in insomnia disorder. Neuroimage Clin 28:102474.

2. Valvassori SS, Dal-Pont GC, Resende WR, Varela RB, LopesBorges J, Cararo JH, Quevedo J (2019) Validation of the animal model of bipolar disorder induced by Ouabain: face, construct and predictive perspectives. Transl Psychiatry 9:158.

3. Mavanji V, Billington CJ, Kotz CM, Teske JA (2012) Sleep and obesity: a focus on animal models. Neurosci Biobehav Rev 36:1015-1029.

4. Calegare BF, Fernandes L, Tufik S, D'Almeida V (2010) Biochemical, biometrical and behavioral changes in male offspring of sleep-deprived mice. Psychoneuroendocrinology 35:775-784.

5. Demet MM, Ozmen B, Deveci A, Boyvada S, Adigüzel H, Aydemir O (2002) Depression and anxiety in hyperthyroidism. Arch Med Res 33:552-556.

6. Fahrenfort JJ, Wilterdink AM, van der Veen EA (2000) Longterm residual complaints and psychosocial sequelae after remission of hyperthyroidism. Psychoneuroendocrinology 25:201-211.

7. Ellenbroek BA, Cools AR (1990) Animal models with con- 
struct validity for schizophrenia. Behav Pharmacol 1:469-490.

8. Fineberg NA, Chamberlain SR, Hollander E, Boulougouris V, Robbins TW (2011) Translational approaches to obsessivecompulsive disorder: from animal models to clinical treatment. Br J Pharmacol 164:1044-1061.

9. Liao Z, Li S, Huang Y, Luo X, Zhong Y, Ji Y, Su D, Ai Z (2020) Metabolite profile changes in different regions of rat brain affected by Ephedra sinica. Evid Based Complement Alternat Med 2020:8358039.

10. Shan B, Ai Z, Zeng S, Song Y, Song J, Zeng Q, Liao Z, Wang T, Huang C, Su D (2020) Gut microbiome-derived lactate promotes to anxiety-like behaviors through GPR81 receptormediated lipid metabolism pathway. Psychoneuroendocrinology 117:104699.

11. Song Y, Shan B, Zeng S, Zhang J, Jin C, Liao Z, Wang T, Zeng Q, He H, Wei F, Ai Z, Su D (2021) Raw and wine processed Schisandra chinensis attenuate anxiety like behavior via modulating gut microbiota and lipid metabolism pathway. J Ethnopharmacol 266:113426.

12. Provini F, Lombardi C, Lugaresi E (2005) Insomnia in neurological diseases. Semin Neurol 25:81-89.

13. Bonnet MH, Arand DL (1997) Hyperarousal and insomnia. Sleep Med Rev 1:97-108.

14. Bonnet MH, Arand DL (2010) Hyperarousal and insomnia: state of the science. Sleep Med Rev 14:9-15.

15. Riemann D, Spiegelhalder K, Feige B, Voderholzer U, Berger M, Perlis M, Nissen C (2010) The hyperarousal model of insomnia: a review of the concept and its evidence. Sleep Med Rev 14:19-31.

16. Zhang J, Lam SP, Li SX, Ma RC, Kong AP, Chan MH, Ho CS, Li AM, Wing YK (2014) A community-based study on the association between insomnia and hypothalamic-pituitaryadrenal axis: sex and pubertal influences. J Clin Endocrinol Metab 99:2277-2287.

17. Hackett RA, Dal Z, Steptoe A (2020) The relationship between sleep problems and cortisol in people with type 2 diabetes. Psychoneuroendocrinology 117:104688.

18. Seelig E, Keller U, Klarhöfer M, Scheffler K, Brand S, Holsboer-Trachsler E, Hatzinger M, Bilz S (2013) Neuroendocrine regulation and metabolism of glucose and lipids in primary chronic insomnia: a prospective case-control study. PLoS One 8:e61780.

19. Farina B, Dittoni S, Colicchio S, Testani E, Losurdo A, Gnoni V, Di Blasi C, Brunetti R, Contardi A, Mazza S, Della Marca G (2014) Heart rate and heart rate variability modification in chronic insomnia patients. Behav Sleep Med 12:290-306.

20. Spiegelhalder K, Fuchs L, Ladwig J, Kyle SD, Nissen C, Voder- holzer U, Feige B, Riemann D (2011) Heart rate and heart rate variability in subjectively reported insomnia. J Sleep Res 20(1 Pt 2):137-145.

21. Javaheri S, Redline S (2017) Insomnia and risk of cardiovascular disease. Chest 152:435-444.

22. Ban HJ, Kim SC, Seo J, Kang HB, Choi JK (2011) Genetic and metabolic characterization of insomnia. PLoS One 6:e18455.

23. Winkelman JW, Buxton OM, Jensen JE, Benson KL, O'Connor SP, Wang W, Renshaw PF (2008) Reduced brain GABA in primary insomnia: preliminary data from $4 \mathrm{~T}$ proton magnetic resonance spectroscopy (1H-MRS). Sleep 31:1499-1506.

24. Van Cauter E, Spiegel K, Tasali E, Leproult R (2008) Metabolic consequences of sleep and sleep loss. Sleep Med 9 Suppl 1:S23-S28.

25. Harvey AG (2002) A cognitive model of insomnia. Behav Res Ther 40:869-893.

26. Feige B, Baglioni C, Spiegelhalder K, Hirscher V, Nissen C, Riemann D (2013) The microstructure of sleep in primary insomnia: an overview and extension. Int J Psychophysiol 89:171-180.

27. Bonnet MH, Arand DL (1995) 24-Hour metabolic rate in insomniacs and matched normal sleepers. Sleep 18:581-588.

28. Nofzinger EA, Buysse DJ, Germain A, Price JC, Miewald JM, Kupfer DJ (2004) Functional neuroimaging evidence for hyperarousal in insomnia. Am J Psychiatry 161:2126-2128.

29. Monroe LJ (1967) Psychological and physiological differences between good and poor sleepers. J Abnorm Psychol 72:255-264.

30. Bonnet MH, Arand DL (1997) Physiological activation in patients with Sleep State Misperception. Psychosom Med 59:533-540.

31. Bonnet MH, Arand DL (1998) Heart rate variability in insomniacs and matched normal sleepers. Psychosom Med 60:610-615.

32. Covassin N, de Zambotti M, Sarlo M, De Min Tona G, Sarasso S, Stegagno L (2011) Cognitive performance and cardiovascular markers of hyperarousal in primary insomnia. Int J Psychophysiol 80:79-86.

33. Vgontzas AN, Bixler EO, Lin HM, Prolo P, Mastorakos G, Vela-Bueno A, Kales A, Chrousos GP (2001) Chronic insomnia is associated with nyctohemeral activation of the hypothalamic-pituitary-adrenal axis: clinical implications. J Clin Endocrinol Metab 86:3787-3794.

34. Vgontzas AN, Liao D, Bixler EO, Chrousos GP, Vela-Bueno A (2009) Insomnia with objective short sleep duration is associated with a high risk for hypertension. Sleep 32:491-497. 
35. de Zambotti M, Covassin N, De Min Tona G, Sarlo M, Stegagno L (2011) Sleep onset and cardiovascular activity in primary insomnia. J Sleep Res 20:318-325.

36. Logan RW, McClung CA (2016) Animal models of bipolar mania: the past, present and future. Neuroscience 321:163188.

37. Ashok AH, Marques TR, Jauhar S, Nour MM, Goodwin GM, Young AH, Howes OD (2017) The dopamine hypothesis of bipolar affective disorder: the state of the art and implications for treatment. Mol Psychiatry 22:666-679.

38. Demontis F, Serra F, Serra G (2017) Antidepressant-induced dopamine receptor dysregulation: a valid animal model of manic-depressive illness. Curr Neuropharmacol 15:417-423.

39. Ortiga-Carvalho TM, Chiamolera MI, Pazos-Moura CC, Wondisford FE (2016) Hypothalamus-pituitary-thyroid axis. Compr Physiol 6:1387-1428.

40. Levenson JC, Kay DB, Buysse DJ (2015) The pathophysiology of insomnia. Chest 147:1179-1192.

41. Edinger JD, Bonnet MH, Bootzin RR, Doghramji K, Dorsey CM, Espie CA, Jamieson AO, McCall WV, Morin CM, Stepanski EJ; American Academy of Sleep Medicine Work Group (2004) Derivation of research diagnostic criteria for insomnia: report of an American Academy of Sleep Medicine Work Group. Sleep 27:1567-1596.

42. Schutte-Rodin S, Broch L, Buysse D, Dorsey C, Sateia M (2008) Clinical guideline for the evaluation and management of chronic insomnia in adults. J Clin Sleep Med 4:487-504.

43. Meier-Ewert HK, Ridker PM, Rifai N, Regan MM, Price NJ, Dinges DF, Mullington JM (2004) Effect of sleep loss on Creactive protein, an inflammatory marker of cardiovascular risk. J Am Coll Cardiol 43:678-683.

44. Floam S, Simpson N, Nemeth E, Scott-Sutherland J, Gautam S, Haack M (2015) Sleep characteristics as predictor variables of stress systems markers in insomnia disorder. J Sleep Res 24:296-304.

45. Vgontzas AN, Tsigos C, Bixler EO, Stratakis CA, Zachman K, Kales A, Vela-Bueno A, Chrousos GP (1998) Chronic insomnia and activity of the stress system: a preliminary study. J Psychosom Res 45:21-31.

46. Basta M, Chrousos GP, Vela-Bueno A, Vgontzas AN (2007) Chronic insomnia and stress system. Sleep Med Clin 2:279291.

47. Fisher SP, Foster RG, Peirson SN (2013) The circadian control of sleep. Handb Exp Pharmacol 217:157-183.

48. Baglioni C, Regen W, Teghen A, Spiegelhalder K, Feige B, Nis- sen C, Riemann D (2014) Sleep changes in the disorder of insomnia: a meta-analysis of polysomnographic studies. Sleep Med Rev 18:195-213.

49. Steiger A (2007) Neurochemical regulation of sleep. J Psychiatr Res 41:537-552.

50. Wu HT, Talmon R, Lo YL (2015) Assess sleep stage by modern signal processing techniques. IEEE Trans Biomed Eng 62:1159-1168.

51. Horovitz SG, Fukunaga M, de Zwart JA, van Gelderen P, Fulton SC, Balkin TJ, Duyn JH (2008) Low frequency BOLD fluctuations during resting wakefulness and light sleep: a simultaneous EEG-fMRI study. Hum Brain Mapp 29:671-682.

52. Cervena K, Espa F, Perogamvros L, Perrig S, Merica H, Ibanez $\mathrm{V}$ (2014) Spectral analysis of the sleep onset period in primary insomnia. Clin Neurophysiol 125:979-987.

53. Spiegelhalder K, Regen W, Feige B, Holz J, Piosczyk H, Baglioni C, Riemann D, Nissen C (2012) Increased EEG sigma and beta power during NREM sleep in primary insomnia. Biol Psychol 91:329-333.

54. St-Jean G, Turcotte I, Pérusse AD, Bastien CH (2013) REM and NREM power spectral analysis on two consecutive nights in psychophysiological and paradoxical insomnia sufferers. Int J Psychophysiol 89:181-194.

55. Riemann D, Nissen C, Palagini L, Otte A, Perlis ML, Spiegelhalder K (2015) The neurobiology, investigation, and treatment of chronic insomnia. Lancet Neurol 14:547-558.

56. Saper CB, Chou TC, Scammell TE (2001) The sleep switch: hypothalamic control of sleep and wakefulness. Trends Neurosci 24:726-731.

57. Saper CB, Scammell TE, Lu J (2005) Hypothalamic regulation of sleep and circadian rhythms. Nature 437:1257-1263.

58. Saper CB, Fuller PM, Pedersen NP, Lu J, Scammell TE (2010) Sleep state switching. Neuron 68:1023-1042.

59. Möhler H (2006) GABAA receptors in central nervous system disease: anxiety, epilepsy, and insomnia. J Recept Signal Transduct Res 26:731-740.

60. Verkuyl JM, Hemby SE, Joëls M (2004) Chronic stress attenuates GABAergic inhibition and alters gene expression of parvocellular neurons in rat hypothalamus. Eur J Neurosci 20:1665-1673.

61. Benson C, Mifflin K, Kerr B, Jesudasan SJ, Dursun S, Baker G (2015) Biogenic amines and the amino acids GABA and glutamate: relationships with pain and depression. Mod Trends Pharmacopsychiatry 30:67-79. 